\title{
"Curdling" of Soymilk in Coffee: A Study of the Phase Behaviour of Soymilk Coffee Mixtures
}

\author{
Mairi Brown ${ }^{1}$, Francesca Laitano ${ }^{1,2}$, Calum Williams ${ }^{1}$, Bruce \\ Gibson $^{1}$, Mark Haw ${ }^{1}$, Karen Johnston ${ }^{1}$, and Jan Sefcik ${ }^{1}$ \\ ${ }^{1}$ Department of Chemical and Process Engineering, James Weir \\ Building, University of Strathclyde, 75 Montrose Street, Glasgow G1 \\ 1XJ, United Kingdom. \\ ${ }^{2}$ Department of Information, Modeling, Electronics and System \\ Engineering, (D.I.M.E.S.), University of Calabria, Via P. Bucci, Cubo \\ 39C, 87036, Rende (CS), Italy.
}

April 1, 2019

\begin{abstract}
Soymilk is often observed to "curdle" (become inhomogeneous) and then sediment in coffee, which makes it an undesirable product for consumers. This work investigates the phase behaviour (curdling) of an organic, unsweetened, long-life soymilk in instant coffee solutions for a range of coffee and soymilk concentrations, temperature and $\mathrm{pH}$. The temperature vs soymilk concentration phase diagram exhibits a coexistence curve, separating a two-phase region at high temperature from a single-phase region at low temperature. The phase transformation is reversible and it is possible to recover a single phase mixture from the "curdled" mixture either by cooling the mixture or by increasing the soymilk concentration. Image analysis was used to investigate the time evolution of the phase separation and sedimentation processes. Fourier transformation of the images resulted in peaks corresponding to the growing length scale of the phase separation. The rate of growth of peak intensity over time shows a power law dependence on temperature characteristic of spinodal decomposition. However, the observed separation kinetics are not entirely straightforward to explain with common phase separation models.
\end{abstract}




\section{Introduction}

Switching from animal- to plant-based food sources would significantly alleviate local and global environmental problems to which livestock is a major contributor, including land degradation, climate change, water shortage and loss of biodiversity [10]. For example, in comparison to dairy milk, the production of an equivalent volume of soymilk is much less energy [9] and water intensive [3]. However, one of the challenges of plant-based food alternatives is that they may not have the texture, taste or physicochemical behavior of meat and dairy products. One example of this is the use of soymilk in coffee drinks, where the soymilk is often observed to "curdle" or phase separate into two phases with different soymilk concentrations (see Fig. 1) and the similarity in appearance to spoiled dairy milk makes the product unappealing to drink.

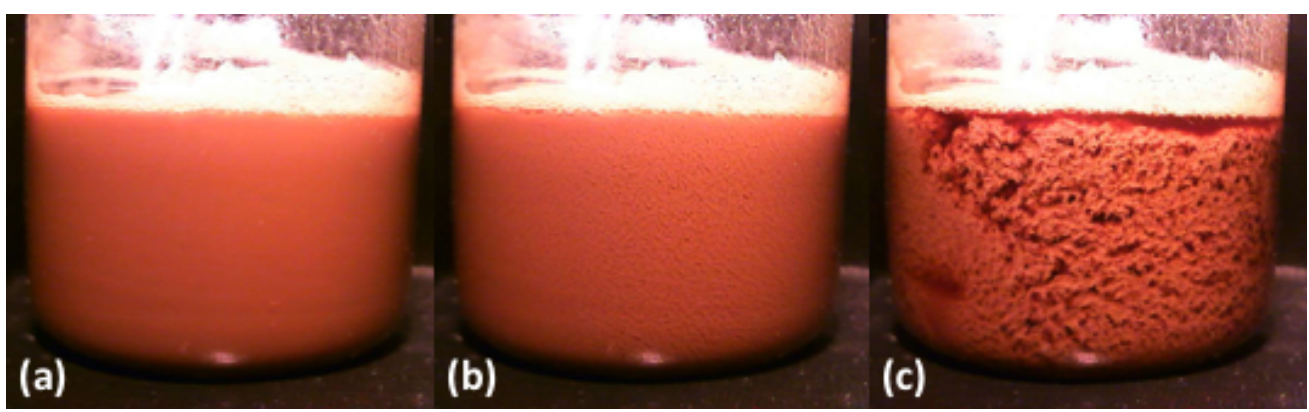

Figure 1: Images of soymilk coffee mixtures in (a) single phase, (b) separating into two phases and (c) sedimentating.

Anecdotal evidence suggests that the curdling behavior is influenced by the concentration of soymilk, the acidity of the coffee, temperature and even the order of adding/mixing the coffee, water and soymilk. There are several brands of soymilk that do not readily exhibit the curdling effect in coffee, and these generally contain acidity regulators and stabilisers, such as monopotassium phosphate and gellan gum, respectively. This suggests that the occurrence of curdling of soymilk in coffee, which involves protein coagulation or aggregation, is influenced by temperature and $\mathrm{pH}$.

While there are numerous studies on isolated soy protein systems, which differ considerably from soymilk, soymilk and soymilk particles have received less attention. Soymilk is a hydrocolloid containing proteins, lipids, minerals and other components, and is much more complex than the soy protein isolate. Soymilk is extracted from whole soybeans and is prepared 
by soaking soybeans in water, wet grinding, heat treatment, and filtration [5]. The lipid, saccharide, and protein components of soybeans are retained in the soymilk [8]. The composition of soymilk varies depending on processing conditions and bean variety but in general contains about $2 \% \mathrm{w} / \mathrm{w}$ lipid, $3-4 \% \mathrm{w} / \mathrm{w}$ protein, and $2 \% \mathrm{w} / \mathrm{w}$ carbohydrate $[5,6,2]$. The main proteins present in soybean are glycinin (11S) and $\beta$-conglycinin $(7 \mathrm{~S})$, which constitutes about $70 \%$ of the total soy protein [4]. The complex behaviour of colloidal systems under various conditions $(\mathrm{pH}$, ionic strength, buffer salts, temperature) is still not well understood and the conditions under which aggregation phenomena occur can vary significantly, depending on the system and the application targeted.

In this work we determine the conditions leading to curdling of soymilk in coffee, and provide insight into the separation and sedimentation mechanisms. A phase diagram for soymilk in coffee is developed for a range of soymilk-coffee-water concentration, temperature and $\mathrm{pH}$. Image analysis is used to quantify the phase separation kinetics during curdling, and thus obtain information on the mechanism of the separation process and its dependence on the location in the phase diagram.

\section{Method}

The experiments used Tesco Gold freeze-dried instant coffee granules, strength 3, and Tesco organic long-life unsweetened soymilk (both from Tesco PLC, UK). The soymilk ingredients were water, organic soy bean $(6 \%)$ and natural flavouring. The composition of $100 \mathrm{ml}$ of soymilk is $1.9 \mathrm{~g}$ of fat, $0.4 \mathrm{~g}$ of carbohydrate, $3.4 \mathrm{~g}$ of protein and $0.1 \mathrm{~g}$ of salt. The jar of coffee granules was stored in the lab at room temperature (approx $21^{\circ} \mathrm{C}$ ) and the soymilk was kept refrigerated once opened.

To prepare the solutions coffee granules were weighed to an accuracy of $0.1 \mathrm{mg}$ and transferred to a $200 \mathrm{ml}$ glass beaker. $100 \mathrm{ml}(100 \mathrm{~g})$ of deionised water was added to the coffee granules and the mixture was stirred until the coffee was dissolved. Soymilk was then added to the coffee solution. Experiments were carried out using coffee to water ratios of $0.0125,0.025$, 0.05 and $0.075 \mathrm{~g}$ coffee/g water, and soymilk concentrations from $2-25 \% \mathrm{w} / \mathrm{w}$. The coffee to water ratios and soymilk concentrations were chosen based on realistic measures typical of those used in preparing coffee drinks.

The $\mathrm{pH}$ of the coffee/water/soymilk mixture was measured using a Jenway $3510 \mathrm{pH}$ meter. After every use of the $\mathrm{pH}$ meter probe, it was rinsed

with distilled water, wiped with a paper towel and placed back into the 
buffer storage solution. The $\mathrm{pH}$ of the pure soymilk was also recorded at the start of every experiment to check for changes over time after opening the carton. We observed that after opening the soymilk carton, the $\mathrm{pH}$ of the soymilk reduced from 7.25 to 7.10 over a period of 10 days, as can be seen in Supplementary Information. A given carton of soymilk was used for no longer than two weeks after opening.

A magnetic pellet was placed into the mixture and the beaker placed onto a Stuart temperature-controlled heat plate and magnetic stirrer. An additional thermocouple, connected to the $\mathrm{pH}$ meter, was placed into the beaker to check the accuracy of the thermocouple on the temperature-controlled heat plate and to provide a temperature correction for the $\mathrm{pH}$ readings. A webcam was used to record images of the mixture. The experimental set up is shown in Supplementary Information.

To obtain the temperature dependent phase behaviour, the mixture was stirred and heated from room temperature to $25^{\circ} \mathrm{C}$, then heated in $5^{\circ} \mathrm{C}$ increments to $60^{\circ} \mathrm{C}$. At and above $40^{\circ} \mathrm{C}$ the beaker was covered with Parafilm to minimise evaporation. Once a temperature increment was reached the webcam imaging was started and the stirrer was switched off. The webcam recorded pictures of the beaker every 5 seconds for 5 minutes. At the end of the 5 minutes the $\mathrm{pH}$ and phase were recorded (single phase, two phase, or "unclear" if separation could not be unambiguously detected by eye).

To obtain information about the phase separation and curdling rate, webcam images were analysed by computing the two-dimensional Fourier transform of the pixel intensity, after cropping an area at the centre of the image and subtracting a reference background image to minimise the effect of fixed optical imperfections such as extraneous reflections. The reference background image was taken while the magnetic stirrer was still switched on. A peak in the radially-averaged Fourier transform magnitude $S(Q)$ at a given wave number magnitude $Q$ essentially identifies significant image intensity variations around the length scale $L=2 \pi / Q[7]$. Hence, for example, separation and coarsening into phases of differing soymilk concentration at a growing length-scale $L$ would be indicated by a peak in $S(Q)$ which grows in intensity and moves to smaller $Q$ over time. The time-dependence of peak position and intensity can then be used to compare the soymilk separation process with common phase separation and flocculation models such as classical spinodal decomposition [12], cluster aggregation [7] and protein cluster formation [11]. 


\section{Results and Discussion}

\subsection{Phase diagrams}

Fig. 2 shows the temperature vs soymilk concentration phase diagrams for a $0.05 \mathrm{~g}$ coffee $/ \mathrm{g}$ water solution. The phase diagrams for different coffee concentrations, namely $0.0125,0.025$ and $0.075 \mathrm{~g}$ coffee/g water, on heating show similar trends (see Supplementary Information). The phase diagram reveals a coexistence curve separating a two phase region at high temperature from a single phase region at low temperature. For low soymilk concentrations, the phase coexistance boundary is high, and then sharply decreases as the concentration increases, reaching a minimum below $25^{\circ} \mathrm{C}$ between 5 $10 \% \mathrm{w} / \mathrm{w}$ soymilk concentration. As the soymilk concentration is further increased, the coexistence temperature increases more slowly. At low con-

centrations the coexistence curve is very sensitive to small fluctuations in mixture composition and temperature.

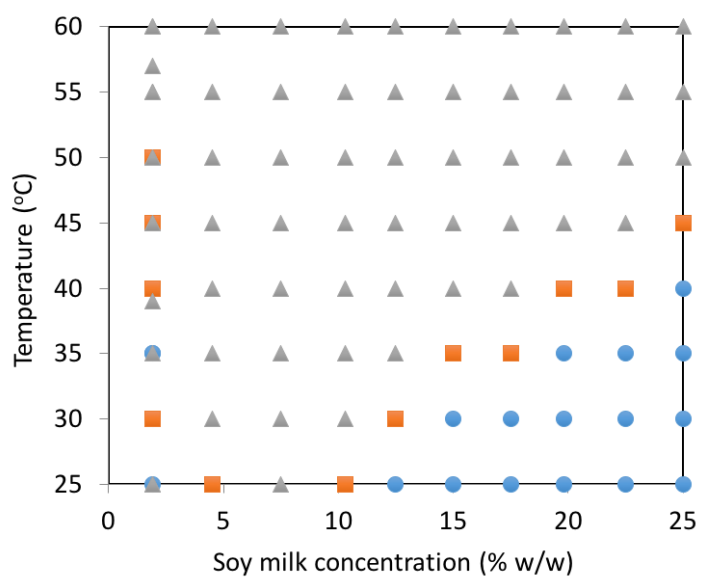

Figure 2: Temperature and soymilk concentration phase diagram for a 0.05 g coffee/g water solution. Blue circles are single phase, grey triangles are two phase, and orange squares show where the phase was unclear.

Interestingly, this phase separation behaviour is reversible, demonstrating that this is a true phase transformation and not an irreversible change due to e.g. protein denaturation. Experiments showed that it is possible to recover the homogeneous mixture in two ways: by cooling or by increasing the soymilk concentration. Mixtures in a two-phase state at high temperature were cooled (maintaining fixed composition) and at low enough tem- 
perature (and after stirring) became homogeneous. In addition, the effect of increasing soymilk concentration in mixtures at constant temperature with a coffee concentration of $0.0125 \mathrm{~g}$ coffee/g water was tested. In these experiments soymilk was gradually added to the mixture, held at either 25, 30,40 or $50^{\circ} \mathrm{C}$, and the resulting phase after monitoring for 5 minutes at each concentration was recorded. It was observed that the mixture was homogeneous at low concentrations (around 2\%), then phase separated at intermediate concentrations, and became homogeneous again at high soymilk concentrations, depending on temperature (see Fig. 2).

The relationship between the phase of the mixture and its $\mathrm{pH}$ was also investigated although there was no clear $\mathrm{pH}$ transition. The phases for $\mathrm{pH}$ vs soymilk concentration and for $\mathrm{pH}$ vs temperature are shown in Supplementary Information. In general, we see that two-phase mixtures have a lower $\mathrm{pH}$ than single phase. However, $\mathrm{pH}$ differences in these mixtures are small.

\subsection{Separation and sedimentation rates}

In this section we explore the time evolution of the curdling and sedimentation processes and the dependence on soymilk concentration and temperature. Fig. 1 shows example images of a mixture with $0.05 \mathrm{~g}$ coffee/g water and a soymilk concentration of $15.0 \% \mathrm{w} / \mathrm{w}$ at $60^{\circ} \mathrm{C}$ that starts as a single phase, separates into a two phase mixture, and subsequently sediments. The images were taken at $5 \mathrm{~s}, 65 \mathrm{~s}$, and $125 \mathrm{~s}$ after stirring was stopped. We note that throughout the phase separation process the flocs can be seen to sink slowly, and at some stage they undergo a rapid collapse, in a behaviour reminiscent of so-called delayed sedimentation [1].

Fig. 3 shows radially averaged Fourier transforms, $S(Q)$, of a fixed $280 \times$ 280 pixels section of images for the example mixture of Fig. 1. One pixel corresponds to approximately $0.08 \mathrm{~mm}$. After $10 \mathrm{~s}$ a shoulder develops, then at $15 \mathrm{~s}$, a peak becomes visible at around $Q \approx 0.6$ inverse pixels, which shifts to smaller $Q$ (larger separation length scale) and grows in intensity (greater contrast between phases) as separation and coarsening proceeds. $S(Q)$ rises monotonically as $Q \rightarrow 0$ and after 115 s the peak disappears, corresponding to the final macroscopic collapse of the suspension. Similar qualitative behaviour is seen for all mixtures at temperatures that undergo separation.

To analyse the time-dependence of the peak position, $Q_{\max }$, and intensity, $S\left(Q_{\max }\right), Q_{\max }$ and $S\left(Q_{\max }\right)$ at each time were estimated by fitting polynomials to the $S(Q)$ curves in the region of the peak. The tempo- 


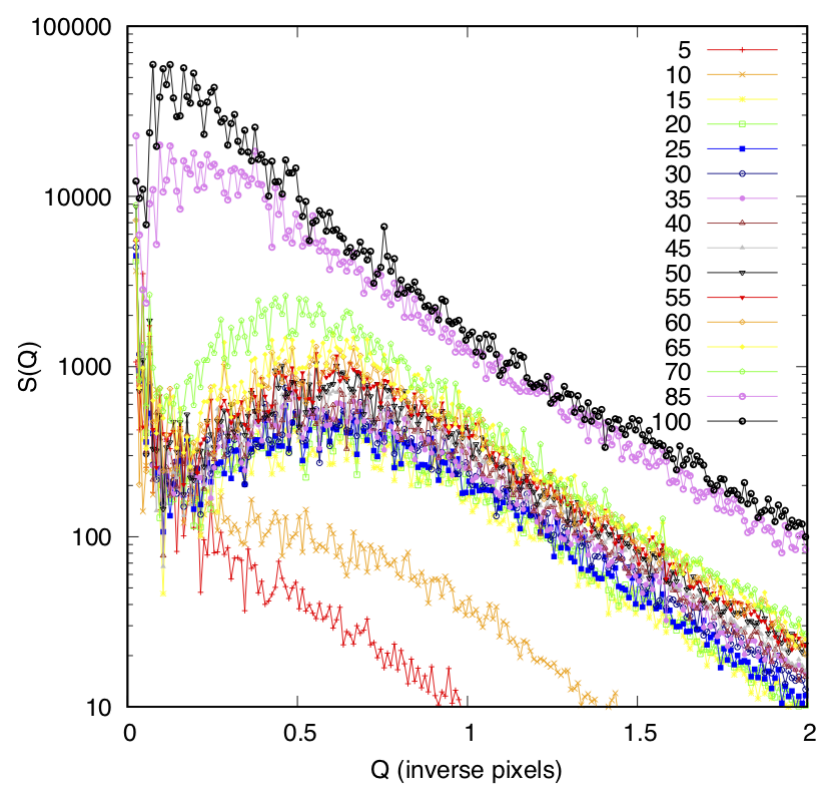

Figure 3: Intensity $S(Q)$ vs $Q$ (in inverse pixels) for Fourier transforms of series of images, taken every $5 \mathrm{~s}$ up to $100 \mathrm{~s}$, for a mixture of $0.05 \mathrm{~g}$ coffee $/ \mathrm{g}$ water and soymilk concentration of $15.0 \% \mathrm{w} / \mathrm{w}$, at a temperature of $60^{\circ} \mathrm{C}$.

ral behavior of the peak position for a mixture with $0.05 \mathrm{~g}$ coffee/g water and soymilk concentration $15.0 \% \mathrm{w} / \mathrm{w}$ at different temperatures is shown in Fig. 4(a). At low temperatures of $25^{\circ} \mathrm{C}$ and $30^{\circ} \mathrm{C}$, there was no separation and hence no peak, as expected from the phase diagram in Fig. 2. At intermediate temperatures of $35^{\circ} \mathrm{C}$ and $40^{\circ} \mathrm{C}$, the mixture partially separates as demonstrated by a clear peak in $S(Q)$, but within the time of the experiment does not undergo sudden collapse. The peak in $S(Q)$ is at an approximately constant position $Q_{\max }$ over a long time period: separation of the phases appears to stabilise at an approximately constant temperaturedependent length scale, although peak intensity (see below) continues to increase implying an increase in the density "contrast" between the phases. The temperature dependence of approximately constant $Q_{\max }$ is consistent with the mixture becoming unstable at a smaller length scale as we go deeper into the two-phase region i.e. at higher temperature.At temperatures of $45^{\circ} \mathrm{C}$ and higher, separation involves a shorter time with approximately constant $Q_{\max }$ followed by rapid collapse, where we see a fast increase in length scale (decrease in $Q_{\max }$ ). The time at which rapid collapse occurs is 
generally shorter for higher temperatures, although measurement of collapse time is subject to uncertainties because collapse was sometimes observed to be position-dependent in the sample vial: images were obtained only from one position but on some occasions the onset of collapse could be observed elsewhere in the vial before becoming visible in the image region. For example, it can be seen from the $S(Q)$ data that the $50^{\circ} \mathrm{C}$ mixture appears to collapse before the $55^{\circ} \mathrm{C}$ mixture, possibly because the images in the $55^{\circ} \mathrm{C}$ case did not capture the earliest instants of collapse. Further study is required to fully characterise the late-stage complete collapse. Comparison with detailed studies of delayed sedimentation in colloidal gels [1] would be interesting.

The temporal behaviour of the peak intensity $S\left(Q_{\max }\right)$ for a mixture with $0.05 \mathrm{~g}$ coffee/g water and soymilk concentration $15.0 \% \mathrm{w} / \mathrm{w}$ at different temperatures is shown in Fig. 4(b). At any given temperature, the intensity increases with time as separation proceeds, with higher temperatures generally giving higher intensities and faster increase. For mixtures at $35^{\circ} \mathrm{C}$ and $40^{\circ} \mathrm{C}$, even though a constant $Q_{\max }$ persists for significant time, $S\left(Q_{\max }\right)$ continues to increase. For higher temperatures, rapid collapse of the mixture coincides with rapid late-time increase of $S\left(Q_{\max }\right)$. In fact, $S\left(Q_{\max }\right)$ increases approximately exponentially over time (before rapid collapse due to sedimentation), with a characteristic time, $\tau$, that decreases as temperature increases: the deeper the sample in the two-phase region, the faster the separation. Plotting characteristic time vs the quench depth, $T-T_{C}$, where we estimate the critical temperature, $T_{C}$, to be $24^{\circ} \mathrm{C}$ from the experimental phase diagram, gives a power law with exponent -2.08 , which is consistent with spinodal models (see below) predicting an exponent of -2 (Fig. 5). Details about the fitting is given in Supplementary Information.

The effect of varying soymilk concentration on the $S(Q)$ peak during separation and collapse at fixed temperature is shown for a temperature of $35^{\circ} \mathrm{C}$ in Fig. 6. From the phase diagram in Fig. 2 we expect to see no separation at soymilk concentrations above $17.5 \% \mathrm{w} / \mathrm{w}$. At concentrations of $4.5 \% \mathrm{w} / \mathrm{w}$ and $7.5 \% \mathrm{w} / \mathrm{w}$, which are near the phase boundary (see Fig. 2) we observe peaks at smaller $Q_{\max }$ compared with higher concentration. Peaks at the higher concentrations of $12.5 \% \mathrm{w} / \mathrm{w}, 15.0 \% \mathrm{w} / \mathrm{w}$ and $17.5 \% \mathrm{w} / \mathrm{w}$ appear to stabilise at a particular $Q_{\max }$ and the samples do not undergo collapse. Intermediate concentrations of $7.5 \% \mathrm{w} / \mathrm{w}$ and $10.3 \% \mathrm{w} / \mathrm{w}$ show separation followed by collapse. The decrease of the typical scale of separation (the inverse of $Q_{\max }$ ) from large scale near the low-concentration phase boundary, to a minimum near the middle of the two-phase region, and subsequent increase again as the high concentration boundary is approached, is con- 

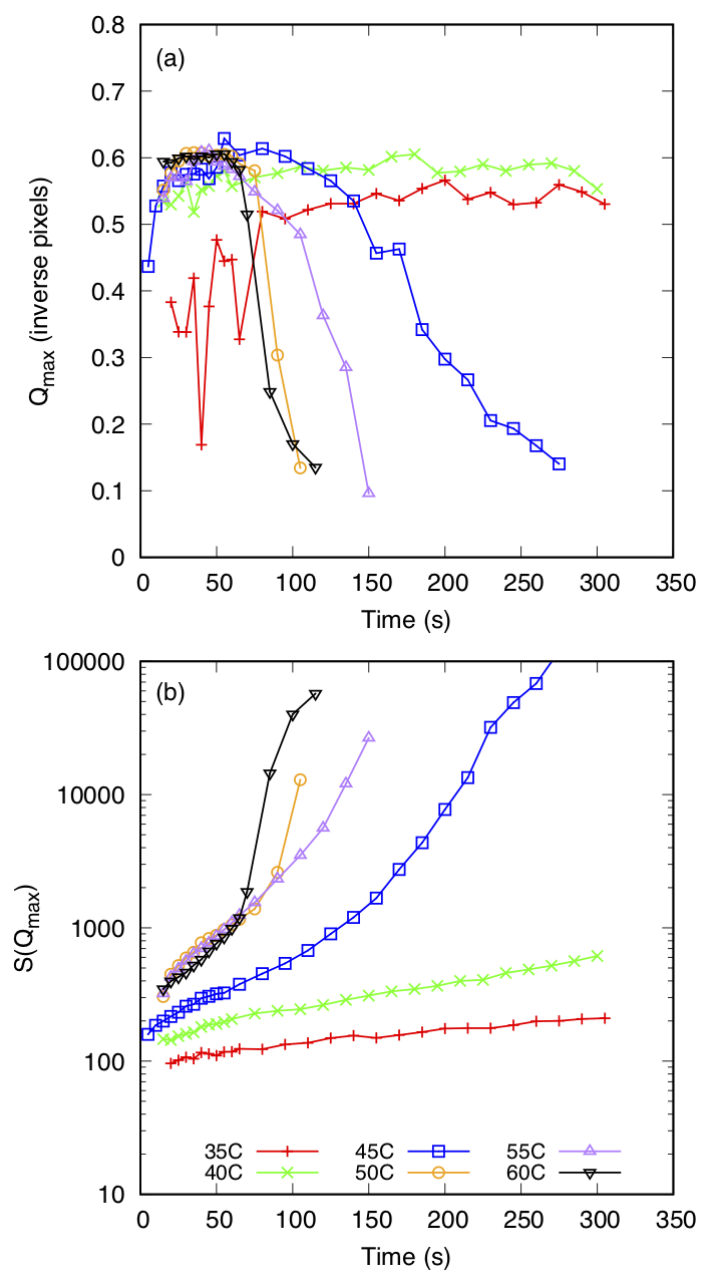

Figure 4: Variation of peak position $Q_{\max }$ (a) and intensity $S\left(Q_{\max }\right)$ (b) with time for different temperatures at a soymilk concentration of $15 \% \mathrm{w} / \mathrm{w}$.

sistent with the relative volumes of the two phases expected (as predicted through a tie-line construction): nearer the phase boundary, a small amount of one of the phases is expected (the dense phase, near the low concentration boundary; the dilute phase, near the high concentration boundary), so that regions of this phase will appear at lower number concentration as separation begins, i.e. dispersed at greater distance from each other. Hence the concentration modulation giving rise to the $S(Q)$ peak is at larger characteristic scale/smaller $Q_{\max }$ near the phase boundaries. Conversely, at maximum 


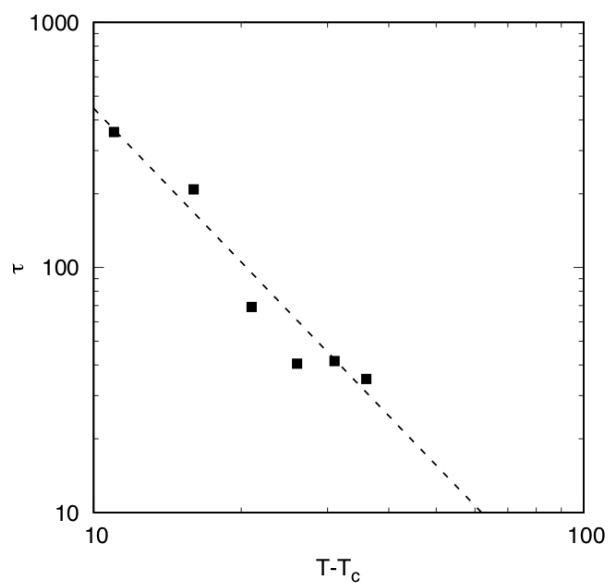

Figure 5: Variation of the characteristic time $\tau$ with the quench depth $T-T_{C}$ showing a power law with exponent -2.08 .

distance from the boundaries, i.e. midway between the boundaries, one expects approximately $50 \%$ by volume of each phase and a maximum number concentration of separating phase regions, i.e. a minimum characteristic scale of density modulation or maximum peak $Q_{\max }$.

The peak intensities $S\left(Q_{\max }\right)$ follow a similar temporal trend as before, increasing until the onset of collapse and rapid sedimentation. Compared to the fixed concentration case, shown in Fig. 4 at different concentrations, the rate of growth in intensity is less clearly exponential but qualitatively the variation with concentration is consistent with the samples' location in the phase diagram:the rate of intensity growth reaches a maximum around the middle of the two-phase region $(7.5 \%$ and $10.3 \% \mathrm{w} / \mathrm{w}$ soymilk) i.e. for the deepest quench into the two-phase region.

Models of spinodal decomposition [12] predict, at the earliest stage of separation, a fastest growing concentration fluctuation at a constant lengthscale given by the characteristic spatial scale at which the mixture is maximally unstable. At first sight this constant length scale matches our results at temperatures/concentrations where $Q_{\max }$ is approximately constant during separation. The exponential growth of peak intensity over time, and the power law dependence of the characteristic growth time on the quench depth, with an exponent close to -2 , are also consistent with expectations from spinodal models. However, such early time spinodal separation is usually expected to rapidly transition to a growing characteristic scale as regions of the two phases coarsen to larger scale (e.g. by coalescence, surface-tension 
driven coarsening, or other mechanisms). Alternative explanations for separation displaying a constant characteristic scale include gelation, where growth is halted by formation of a system-spanning gel phase $[1,7]$ which in many systems then suddenly collapses after a certain delay time due to thermal consolidation, and formation of a 'cluster phase' due to the combination of short-range attraction and longer-range weak repulsion between particles [11]. However, the fact that the peak intensity continues to grow during the constant $Q_{\max }$ period is difficult to explain if growth were really frustrated by either of these mechanisms, unless consolidation and local densification could occur that increased the density contrast without changing the length scale. A study of the dynamics of the particles in this regime, for example by dynamic light scattering, could be used to confirm gelation, although the turbidity of the samples makes this challenging.

In the separating mixtures, especially near the phase boundaries, there is evidence of an increase in peak position at the earliest times (see Figs. 4(a) and 6(a)), implying a decreasing length scale during the initial stages of phase separation. Such a decrease in characteristic scale does not agree with expectations from aggregation and gelation models [7] or general models of diffusion-driven phase separation [12] and seems to effectively contradict coarsening-based models where regions of separating phases should be growing. A possible explanation, suggested by the fact that we observe this for the shallowest quenches into the two-phase region, i.e. near the phase boundaries, is that we are observing ongoing nucleation of droplets at a rate faster than growth of existing nuclei. At the earliest stages of separation as the first droplets of the separating phase nucleate, their number concentration will be low giving a large droplet-to-droplet distance and hence large characteristic scale of concentration modulation i.e. a small $Q_{\max }$. With increasing time more droplets nucleate, increasing the number concentration, decreasing the characteristic distance between droplets and thus increasing $Q_{\max }$. Further experiments with better spatial and time resolution would be useful to explore this nucleation region in more detail: the image quality and time resolution of the experiments described here provides relatively low contrast in the early-time $S(Q)$ peaks, leading to difficulties with precise peak position estimation in some datasets.

\section{Conclusions}

The phase behaviour of soymilk coffee mixtures with temperature, $\mathrm{pH}$, and soymilk and coffee concentrations were investigated. Mixtures of known 

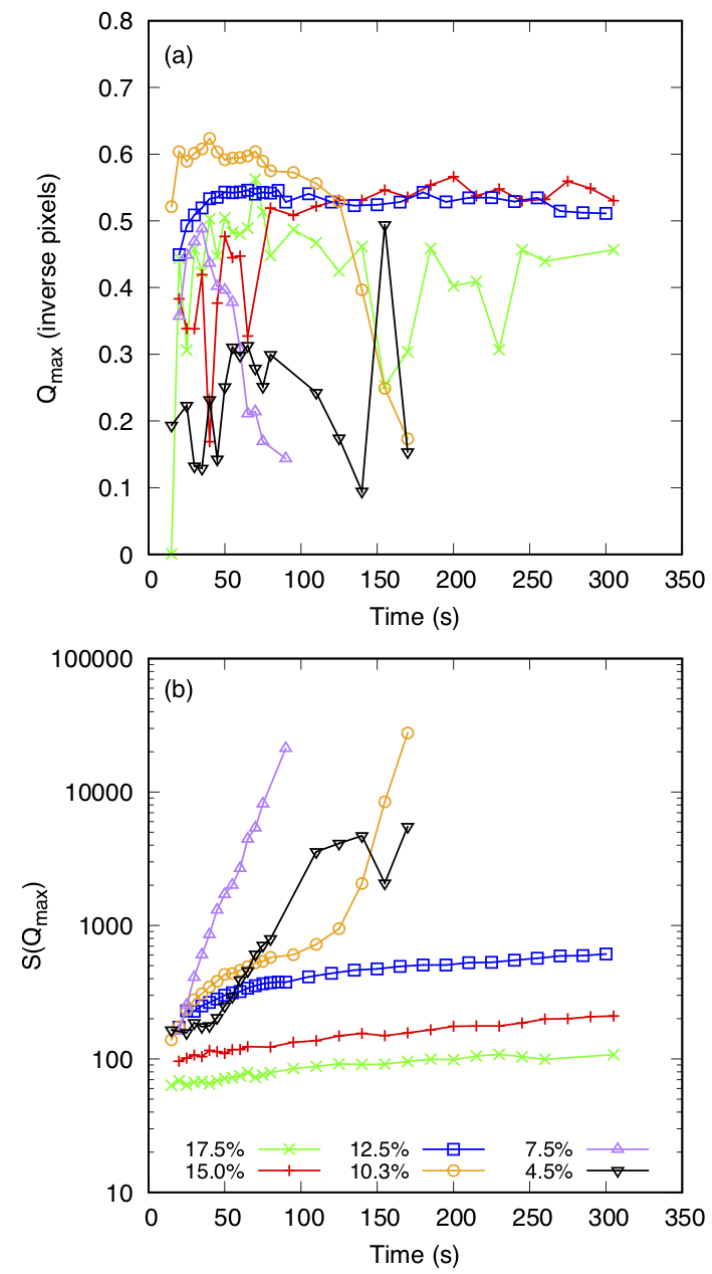

Figure 6: Variation of peak position (a) and intensity (b) with time for different soymilk concentrations at a temperature of $35^{\circ} \mathrm{C}$.

composition were heated to a fixed temperatures and their phase behaviour over a period of five minutes was recorded. In general, the mixtures phase separated at high temperatures and the temperature vs soymilk concentration phase diagram exhibits a coexistence curve, separating a two-phase region at high temperature from a single-phase region at low temperature. The phase transition occurred at higher temperatures for high and low soymilk concentrations and a temperature minimum at intermediate concentrations.

Image analysis was used to explore how the kinetics of separation de- 
pended on position in the phase diagram. At temperatures and soymilk concentrations near the coexistence boundary, the characteristic separation length-scale was found to remain approximately constant for a significant time and rapid sedimentation collapse was not observed over the timescale of our measurements. The measured behaviour of the characteristic length scale during phase separation is partly consistent with spinodal models, although questions remain over this interpretation. Deeper within the twophase region, the period of constant length scale was shorter and subsequent rapid sedimentation and collapse occurred. There was some evidence that at the earliest stages of separation, the characteristic length scale decreases, which may indicate a nucleation mechanism, however more detailed data are required to verify and explore this regime.

The chemistry of phase separation in soymilk systems is not well understood. Our measurements, while partly consistent with well-established separation models such as spinodal decomposition and nucleation, indicate significant complexities, which is not surprising given the interplay of the protein physics and chemistry involved in soymilk stability. We note that some types/brands of soy milk are less prone to curdling in coffee, which is likely due to the addition of stabilisers. Further work on the chemistry and physics of phase behavior in soymilk, and other important dairy alternatives such as almond or oat milk, would be of significant predictive and product-development value in the food and drink industry. In particular, the reversibility of the soymilk phase separation could be a useful property to exploit in food product engineering.

\section{Acknowledgements}

We would like to thank BP for sponsorship of Bruce Gibson's summer internship, the University of Calabria for funding Francesca Laitano's exchange visit, and Stephen Euston for valuable discussions.

\section{Supplementary Information}

Electronic Supplementary Information (ESI) available: Images for soymilk coffee mixtures at different concentrations and temperatures were taken every 5 seconds over a period of 5 minutes and are available online. See DOI: 10.15129/95c83586-ab45-4f7b-83f5-b559cf368e08 


\section{References}

[1] P. Bartlett, L. J. Teece, and M. A. Faers. Sudden collapse of a colloidal gel. Phys. Rev. E, 85:021404, 2012.

[2] N. Cruz, M. Capellas, M. Hernandez, A. J. Trujillo, B. Guamis, and V. Ferragut. Ultra high pressure homogenization of soymilk: Microbiological, physicochemical and microstructural characteristics. Food Research International, 40:725, 2007.

[3] A. E. Ercin, M. M Aldaya, and A. Y. Hoekstra. The water footprint of soy milk and soy burger and equivalent animal products. Value of Water Research Report Series, 49, 2011.

[4] Stephen R. Euston, Al-Amari Al-Bakkush, and Lydia Campbell. Comparing the heat stability of soya protein and milk whey protein emulsions. Food hydrocolloids, 23:2485, 2009.

[5] S. K. Giri and S. Mangaraj. Processing Influences on Composition and Quality Attributes of Soymilk and its Powder. Food Eng. Rev., 4:149, 2012 .

[6] Chinyere I. Iwuoha and Kenneth E. Umunnakwe. Chemical, physical and sensory characteristics of soymilk as affected by processing method, temperature and duration of storage. Food Chemistry, 59:373, 1997.

[7] W. C. K. Poon and M. D. Haw. Mesoscopic structure formation in colloidal aggregation and gelation. Advances Interface Colloid Science, 73:71, 1997.

[8] Guo Shun-Tang, Tomotada Ono, and Masayuki Mikami. Interaction between Protein and Lipid in Soybean Milk at Elevated Temperature. J. Agric. Food Chem., 45:4601, 1997.

[9] Annika Smedman, Helena Lindmark-Mansson, Adam Drewnowski, and Anna-Karin Modin Edman. Nutrient density of beverages in relation to climate impact. Food and Nutrition Research, 54:5170, 2010.

[10] H. Steinfeld. Livestock's long shadow: environmental issues and options. Rome: Food and Agriculture Organization of the United Nations, 2006 . 
[11] Anna Stradner, Helen Sedgwick, Frederic Cardinaux, Wilson C. K. Poon, Stefan U. Egelhaaf, and Peter Schurtenberger. Equilibrium cluster formation in concentrated protein solutions and colloids. Nature, 432:492, 2004.

[12] H. Tanaka. Viscoelastic phase separation. J. Phys.: Condensed Matter, 12:R207, 2000. 


\title{
"Curdling" of Soymilk in Coffee: A Study of the Phase Behaviour of Soymilk Coffee Mixtures
}

\author{
Mairi Brown ${ }^{1}$, Francesca Laitano ${ }^{1,2}$, Calum Williams ${ }^{1}$, Bruce \\ Gibson $^{1}$, Mark Haw ${ }^{1}$, Karen Johnston ${ }^{1}$, and Jan Sefcik ${ }^{1}$ \\ ${ }^{1}$ Department of Chemical and Process Engineering, James Weir \\ Building, University of Strathclyde, 75 Montrose Street, Glasgow G1 \\ 1XJ, United Kingdom. \\ ${ }^{2}$ Department of Information, Modeling, Electronics and System \\ Engineering, (D.I.M.E.S.), University of Calabria, Via P. Bucci, Cubo \\ 39C, 87036, Rende (CS), Italy.
}

March 29, 2019

\section{Supplementary Information}

\section{Soymilk ingredients and composition}

Ingredients: Water, Organic Soya Beans (6\%), Natural Flavouring. $100 \mathrm{ml}$ of soymilk contains: Fat $1.9 \mathrm{~g}$, Carbohydrate $0.4 \mathrm{~g}$, Protein $3.4 \mathrm{~g}$, Salt $0.1 \mathrm{~g}$

\section{Experimental setup}

Fig. 1 shows the Stuart magnetic stirrer heating plate with a beaker on top. An additional thermocouple, which was connected to the Jenway $3510 \mathrm{pH}$ meter, was also placed into the beaker. The webcam is on the lower right of the image and a lamp and black background were used to improve image quality and reduce glare.

\section{$\mathrm{pH}$ vs time of soymilk}

The $\mathrm{pH}$ of soya milk was found to slightly decrease with the time after first opening as shown in Fig. 2. 


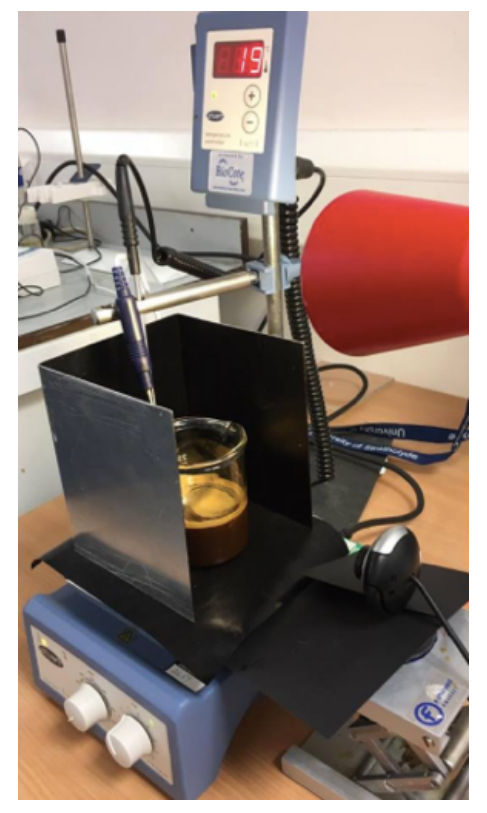

Figure 1: Experimental set up showing a beaker on the temperaturecontrolled magnetic stirring heat plate.

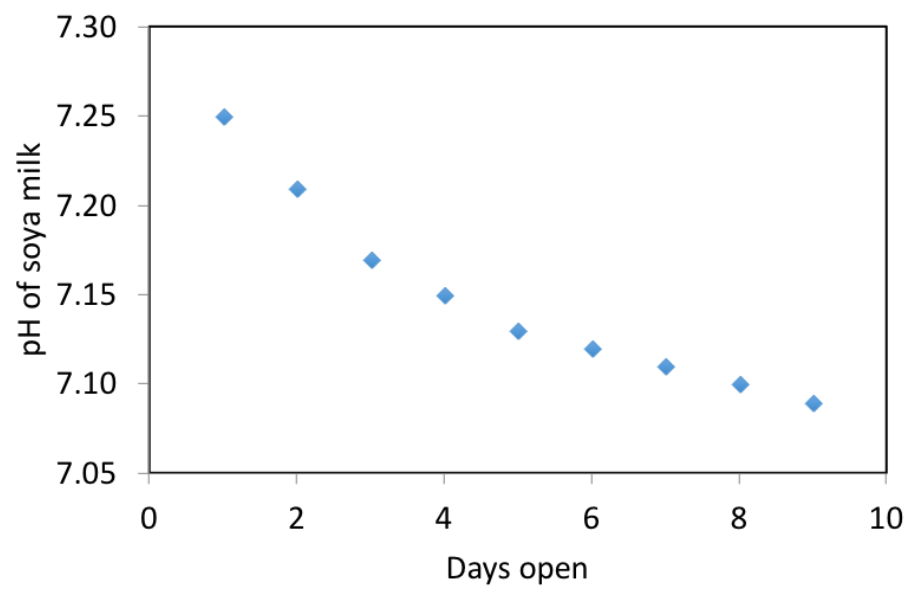

Figure 2: The $\mathrm{pH}$ of the soya milk decreases with time after the pack is first opened.

\section{Phase diagrams for coffee soymilk mixtures}

The phase diagrams in Fig. 3 generally show a coexistence curve separating a two phase region at high temperature from a single phase region at low 
temperature. For the $0.125 \mathrm{~g}$ coffee/g water mixture and soya milk concentrations of below $5 \% \mathrm{w} / \mathrm{w}$ the coexistence curve sharply decreases and reaches a minimum below $25 \mathrm{oC}$ between $5-10 \% \mathrm{w} / \mathrm{w}$ soya milk concentration before increasing more slowly for higher concentrations. The location of the coexistence curve is very sensitive to small fluctuations in mixture composition and temperature. The phase diagrams for the $0.0125,0.025$ and 0.075 g coffee/g water solutions (Fig. 3) show similar trends.
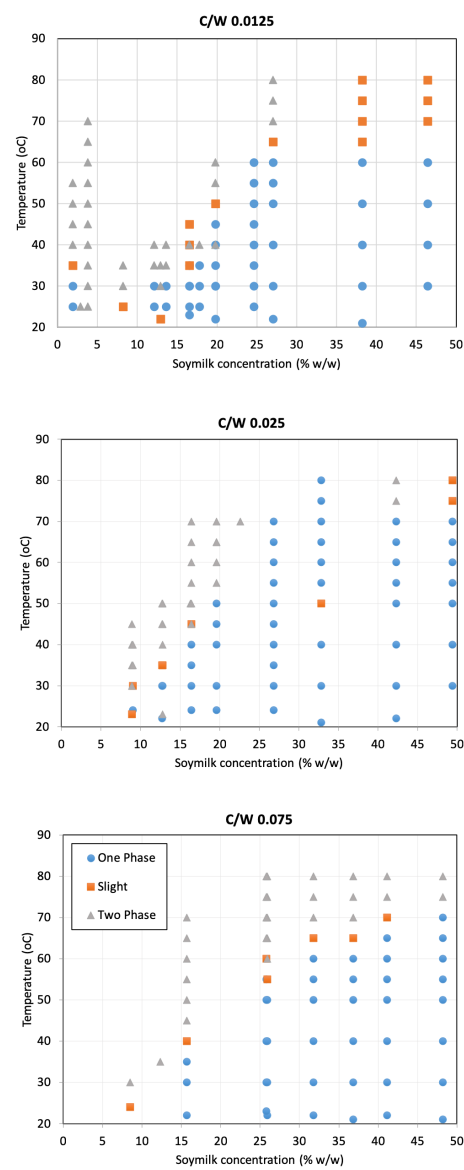

Figure 3: Temperature vs soya milk concentration phase diagrams for coffee water concentrations of $0.025,0.075$ and $0.125 \mathrm{~g}$ coffee/g water, respectively. 


\section{Phase behaviour of soymilk coffee mixtures with $\mathrm{pH}$}

Fig. 4 shows the phase diagram for a coffee water ratio of $0.05 \mathrm{~g}$ coffee/g water as a function of the measured $\mathrm{pH}$ and soymilk concentration. The $\mathrm{pH}$ of all mixtures was within the range of 5.0 to 5.4 and the $\mathrm{pH}$ range is narrow for higher soymilk concentrations but fluctuates more at lower soymilk concentrations. In general, for a fixed soymilk concentration the mixture is two phase at lower $\mathrm{pH}$ and single phase at a higher $\mathrm{pH}$. However, for soymilk concentrations below $5 \% \mathrm{w} / \mathrm{w}$ the behaviour is less well defined. There is no clear $\mathrm{pH}$ value where a transition from single to two phase occurs.

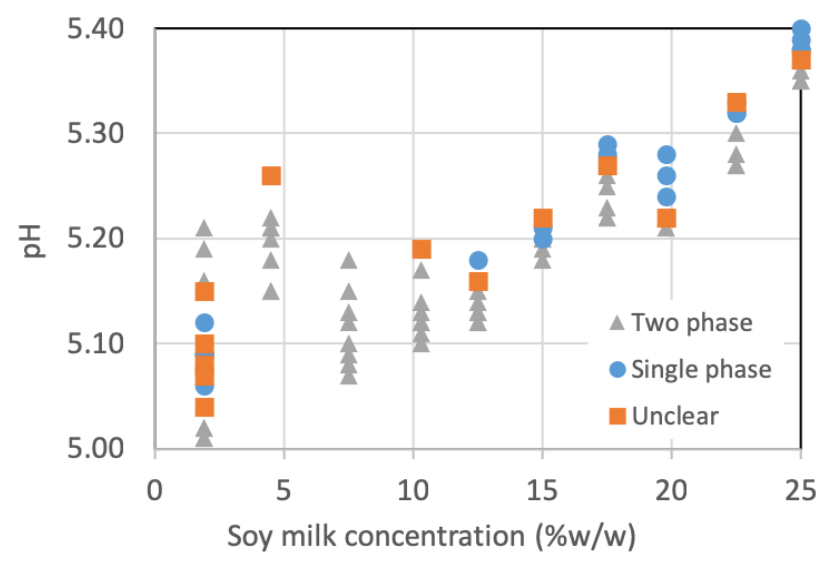

Figure 4: $\mathrm{pH}$ and soymilk concentration phase diagram for $0.05 \mathrm{~g}$ coffee/g water from $25^{\circ} \mathrm{C}$ to $60^{\circ} \mathrm{C}$. Blue circles are single phase, grey triangles are two phase and orange squares show where the phase was unclear.

The variation of $\mathrm{pH}$ was measured during heating and is shown in Fig. 5. The $\mathrm{pH}$ shows a decrease on heating, which is more significant for low soymilk concentrations. The largest change in $\mathrm{pH}$ was observed in the 4.5 $\% \mathrm{w} / \mathrm{w}$ soymilk mixture where the $\mathrm{pH}$ decreased by 0.24 .

The effect of temperature and $\mathrm{pH}$ on the phase behaviour of the system could be due to changes in solubility of the protein molecules, or aggregation of protein molecules. A study of solubility of heat-treated and unheated soy protein isolate dispersions found that soy protein became insoluble as the pH was lowered to around 5 (adjusted using $\mathrm{HCl}$ and $\mathrm{NaOH}$ ) [3]. However, on addition of $\mathrm{NaCl}$ the solubility above $\mathrm{pH}$ values of 5 was decreased, and the heat-treated dispersion showed lower solubility than the unheated 

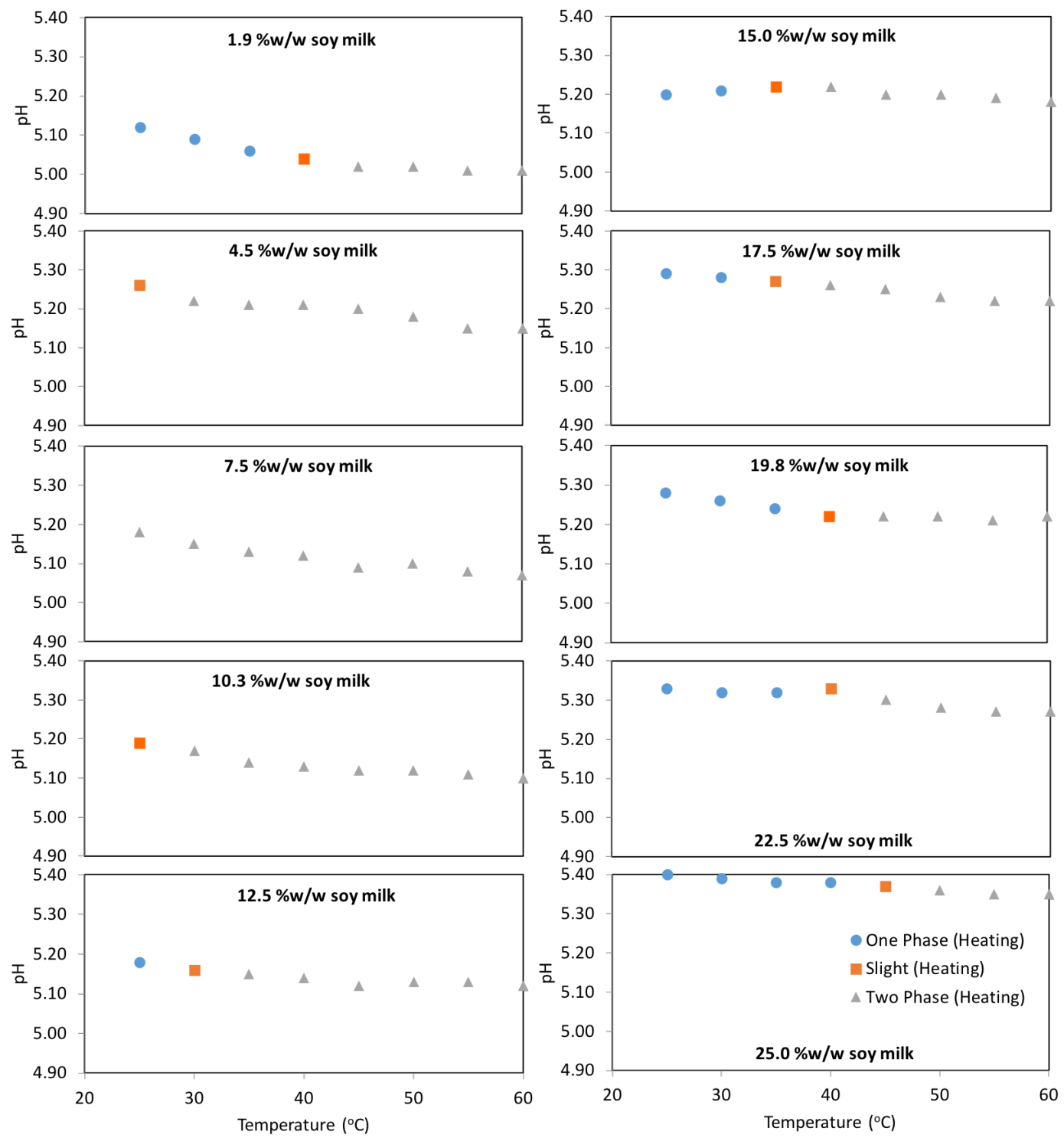

Figure 5: $\mathrm{pH}$ vs temperature for $0.05 \mathrm{~g}$ coffee/g water and different soymilk concentrations. The legend in the bottom right graph applies to all the graphs. 
dispersion. A study of glycinin found that at $20^{\circ} \mathrm{C}$ and ionic strength of 0.5 , glycinin solubility decreases from $100 \%$ to $30 \%$ when the $\mathrm{pH}$ is lowered from 6.5 to 2.5 [2]. For lower ionic strengths there is a sharper decrease in solubility, with $95 \%$ precipitation from $\mathrm{pH} 5.8$ to 4.7 at ionic strength of 0.2 , and complete precipitation from 6.2 to 4.7 , at ionic strength 0.03 . A study on heat stability of soy protein found that aggregation kinetics of emulsion droplets was highest for a $\mathrm{pH}$ between 4.5 and 6 [1]. The $\mathrm{pH}$ range found in these studies for low solubility and aggregation is comparable to the $\mathrm{pH}$ of soymilk coffee mixtures. However, in the case of coffee mixtures, the $\mathrm{pH}$ changes are very small and there is no clear relation between $\mathrm{pH}$ and curdling.

\section{Fitting data}

The data for $S\left(Q_{\max }\right)$ vs time for a soymilk concentration of $15 \% \mathrm{w} / \mathrm{w}$ was fitted using exponential functions of the form $y=A e^{B t}$, as shown in Fig. 6. The exponents, $B$, for each temperature are given in Table 1 . The

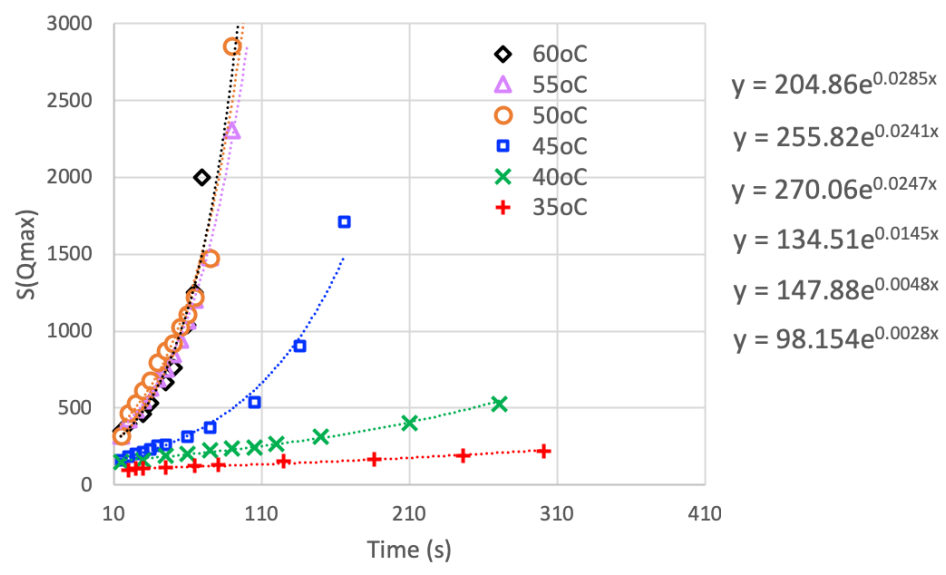

Figure 6: Exponential fits for $S\left(Q_{\max }\right)$ vs time. The equations for the fits are on the right of the chart.

characteristic time is $\tau=\frac{1}{B}$. Based on the phase diagram, we estimated the critical temperature (minimum temperature where the phase transition occurs) to be $24^{\circ} \mathrm{C}$. The resulting power law fit through the data in Table 1 is $\tau=a\left(T-T_{c}\right)^{-b}$, where $a=54114 \mathrm{~s} \mathrm{~K}^{-1}$ and $b=2.08$. 
Table 1: Exponents for each time for the $15 \% \mathrm{w} / \mathrm{w}$ soymilk mixtures.

\begin{tabular}{rr}
\hline$T\left({ }^{\circ} \mathrm{C}\right)$ & $B\left(\mathrm{~s}^{-1}\right)$ \\
\hline 35 & 0.0028 \\
40 & 0.0048 \\
45 & 0.0145 \\
50 & 0.0247 \\
55 & 0.0241 \\
60 & 0.0285 \\
\hline
\end{tabular}

\section{References}

[1] Stephen R. Euston, Al-Amari Al-Bakkush, and Lydia Campbell. Comparing the heat stability of soya protein and milk whey protein emulsions. Food hydrocolloids, 23:2485, 2009.

[2] Cartiona M. M. Lakemond, Harmen H. J. de Jongh, Martin Hessing, Harry Gruppen, and Alphons G. J. Voragen. Heat Denaturation of Soy Glycinin: Influence of $\mathrm{pH}$ and Ionic Strength on Molecular Structure. J. Agric. Food Chem., 48:1991, 2000.

[3] Jacoba M. S. Renkema, Harry Gruppen, and Ton van Vliet. Influence of $\mathrm{pH}$ and Ionic Strength on Heat-Induced Formation and Rheological Properties of Soy Protein Gels in Relation to Denaturation and Their Protein Compositions. J. Agric. Food Chem., 50:6064, 2002. 